\title{
Functioning of Family System in Pediatric Oncology During Treatment Phase
}

\author{
Giovanna Perricone, ${ }^{1}$ Concetta Polizzi, ${ }^{1}$ Maria Regina Morales, ${ }^{1}$ \\ Santo Marino, ${ }^{2}$ and Cinzia Favara Scacco ${ }^{2}$ \\ ${ }^{1}$ Department of Psychology, University of Palermo, Viale Delle Scienze, Palermo, Italy; \\ 2Operating Unit of Pediatric Hematology, University Hospital "G. Rodolico", Via Santa Sofia, \\ Catania, Italy
}

The study focuses on parents' psychological implications caused by the treatment of their children suffering from tumor. It investigates some specific mothers' resource factors such as their strategies of coping and the perception of their own family functioning in terms of cohesion and adaptability.

The study was performed with 34 mothers of children suffering from acute lymphoblastic leukemia (ALL), during the treatment phase. The used tools were the Coping Orientation to Problem Experienced-New Italian Version, to investigate coping strategies, and the Family Adaptability and Cohesion Evaluation Scale-III, to analyze both real and ideal perception of family functioning.

The data related to coping, show how the involved mothers tend to mainly use the strategies of positive aptitude, orientation toward problem and social support $(F=99.88, \mathrm{~d} f=4, P<.01)$. The family functioning, in terms of adaptability, is described as chaotic relating to both the real $\left(\chi^{2}=\right.$ $13.29, \mathrm{~d} f=3, P=.004)$ and ideal $\left(\chi^{2}=11.52, \mathrm{~d} f=2, P=.003\right)$ family, whereas in terms of cohesion, it is perceived as chiefly disengaged in the real family $\left(\chi^{2}=12.3, \mathrm{~d} f=3, P=.006\right)$ and as enmeshed in the ideal one $\left(\chi^{2}=12.58, \mathrm{~d} f=3, P=.006\right)$.

Statistically positive correlations were only detected between adaptability and avoidance $(r=$ $0.49, P<.01)$; adaptability and orientation toward problem $(r=0.36, P<.05)$; and adaptability and transcendent orientation $(r=-0.04, P<.05)$.

Despite the critical situation, the mothers have shown optimistic view, care for problem management and capability to ask for help. These coping strategies allow the therapeutic alliance between families and health care workers, so useful for the quality of childcare.

Keywords compliance, coping behavior, family, pediatric oncology, psychology/psychiatry, quality of life/psychosocial, treatment

Received 3 February 2012; accepted 16 May 2012.

We are grateful to all mothers who were involved in the research. We also thank all health workers of the operating unit of pediatric hematology-University Hospital "G. Rodolico", Catania, who made the work of researchers easier. Moreover, we would like to thank Germana Anzalone, for translation in english.

Address correspondence to Giovanna Perricone, Department of Psychology, University of Palermo, Viale Delle Scienze, ed. 15 - 90128 Palermo, Italy. E-mail: giovanna.perricone@unipa.it 


\section{INTRODUCTION}

Several studies [1-4] display how tumor diagnoses of children, such as the acute lymphoblastic leukemia (ALL), are emotional and cognitive shocks for parents, who have to face and adjust themselves to such a stress factor. It is an acute stress that could become chronic on starting the treatment, especially in mothers [5] who are, typically, the parents mostly involved in facing the collateral effects of clinical treatments, managing various family/home activities, and reorganizing the everyday family tasks and rhythms [6]. Parents' capability of facing the critical event gets weaken during the early phase of the treatment, which is the acute phase of children's pathology [6]. It is a stressor condition that parents live as a sort of surrealistic experience [7]. Most of them perceive their everyday times and spaces as if they were the same of those of the treatment. Such identification reduces, or alters, their ability of facing difficulties, their relational modalities and normal life style. These impairments would prevent parents from building the therapeutic alliance, the synergic relation between cure and care so functional to child's life style $[8,9]$.

Parents, in particular mothers, have to cope with a reality that is unacceptable [10], facing difficulties and changes, adjusting themselves to the event, and looking for a new own developmental balance.

In the light of such considerations, the study is aimed at focusing some aspects such as, in particular, coping strategies and perception of family functioning, which are proved to be important predictive factors related to parents' adjustment to children's tumor [11]. It would seem that such variables are likely moderators that may positively affect parents' adjustment modalities to developmental condition. In fact, the activation of strategies that allow parents to meet the requests of the new situation $[2,12]$, as well as the perception of family support and cohesion $[13,14]$, are mostly linked to positive and adaptive outcomes.

Hence, this study, as a progression from previous other contributions of the field, wants to explore such factors within a group of mothers of children suffering from ALL, diagnosed no later than two months from the start of the treatment. However, unlikely the above-mentioned studies, it also wants to consider the likely correlations of its dimensions in order to start new reflections.

Therefore, the study focuses on the configuration of family system, during the treatment phase, with regards to the relation between the parents' coping strategies, and the perception of their family functioning.

Namely, the explored coping strategies were:

- the social support, as mothers' request for empathic concern to be shown by their partners or any other family member; as search for information about their child's condition; as "emotional outpouring". Such a support may be effective in diminishing the psychological stress levels related to the critical event [15];

- the avoidance, as the negation (within limited time) of the situation or as mental detachment [16];

- the positive aptitude, as the acceptation of the situation and positive reinterpretation of the event leading to optimistic aptitude [7, 17, 18];

- the orientation toward problem, as the skill of eliminating distractive activity to enhance the management of the situation and develop empowerment to control the situation;

- the transcendent orientation as request for help and seeking solace in faith or spirituality, which may convey positive thoughts and self-confidence [19, 20]. 
The other focus of the study, which is the mothers' perception of family functioning, has been studied from the real and ideal family point of view. Specifically, the study deals with two factors of the family functioning:

- the cohesion degree, as the ability of family members of being empathetic and cooperative to reach common goals;

- the adaptability/flexibility degree, as the ability of family system to activate changes in leadership, roles, and relational rules to face the new demands of the treatment phase [21].

Medium levels of cohesion and adaptability make families supportive of each member.

Therefore, considering these two focus fields, the study is a reflection on the likely internal (coping strategies) and external (family functioning) protection factors of the mothers of children suffering from tumor. The protection factors, in fact, allow them to take part easily in their child's cure and care process and to share the goal of the child's tumor recovery with medical staff, and controlling the negative medical and psychosocial effects [9].

\section{MATERIALS AND METHODS}

The study was accomplished in accordance with the ethical research guidelines, fixed by the Declaration of Helsinki in 1964. The informed consent of participants was obtained after that oral information about the study protocol was provided.

The research was performed over a six-month period (June to November 2011), by means of a sample of 34 mothers (mean age 35 years old, $S D=5$ ) of children suffering from ALL, at the early stage of treatment after the disclosure of the diagnosis (Table 1).

The mothers were collected at the Sicilian Pediatric Hematology and Oncology Centre, according to precise criteria, set by the research project, such as Italian nationality of mother and her whole family; leukemia sufferers aged from 6 to 15; disclosure of the diagnosis not sooner than one month and not later than three months; no severe neurologic series and genetic syndromes. Therefore, according to such criteria of eligibility, the sample of the study $(n=34)$ is the result of the selection (see Fig. 1), performed among 45 mothers of children with leukemia.

The hypotheses of the study were:

TABLE 1 Characteristics of the Sample

\begin{tabular}{ll}
\hline Mothers' age & $\begin{array}{l}\text { Mean }=35 \text { years } \\
\text { SD }=5 \text { years } \\
\text { Socio-cultural level (parental educational attainment) }\end{array}$ \\
Children's age & $\begin{array}{c}\text { Medium (about 13 years of } \\
\text { education } \\
M=10 \text { years }\end{array}$ \\
Children's gender & SD $=2$ years \\
Tumor of children: Leukemia & Male $=20$ \\
Phase of leukemia: acute = Start of treatment and first & Female $=14$ \\
hospitalization & $N=34$ \\
Treatment: Chemiotherapy & $N=34$ \\
Time period from the diagnosis up to the start of the research & \\
& $N=34$ \\
& $M=48$ days \\
& $\mathrm{SD}=6$ days \\
\hline
\end{tabular}




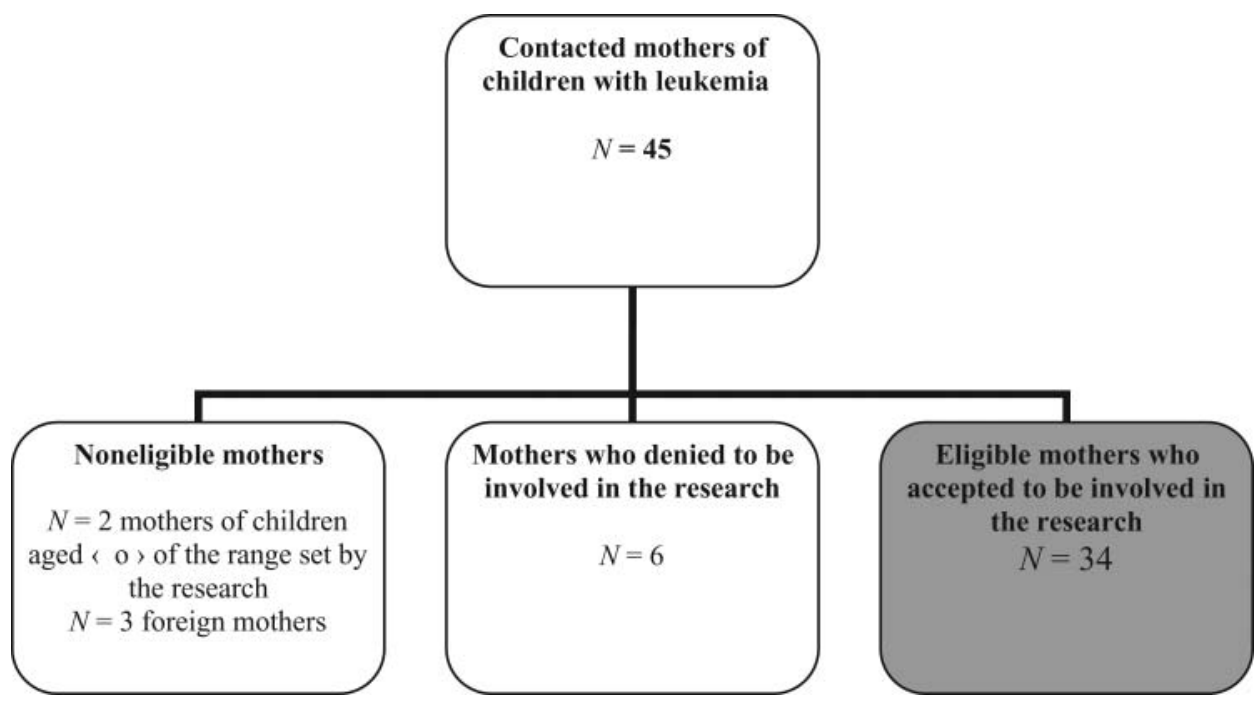

FIGURE 1 Recruitment process of the sample.

- verifying what coping strategies were more frequently used by the mothers to manage the critical events of the treatment phase.

- verifying whether the mothers perceived their own family functioning in terms of cohesion and adaptability.

- verifying the existence of likely correlations between typologies of the mothers' coping strategies, and the indicators of their perception of their own family functioning.

\section{Measures and Procedures}

The administration of two specific tools was planned to verify the hypotheses:

- the COPE-NVI (Coping Orientation to Problem Experienced-New Italian Version) [22], to detect coping strategies activated by mothers in order to cope the stress condition caused by the treatment phase of their child. It is a standardized and validated questionnaire, used to assess five specific coping strategies, such as the social support, avoidance, positive aptitude, orientation toward problem, and transcendent orientation. The internal structure of the tool, whose original version [23] has been translated into Italian and validated on a sample made up of 458 Italian subjects (mean age $=49.2, \mathrm{SD}=16.2$ ), was obtained through a factorial analysis that detected the earlier-mentioned five factors. The questionnaire has good internal consistency (Cronbach's alpha $>$.70; coefficients of correlation among different items-total $r>0.30$ ), and good test-retest reliability (test-retest $=/>.70$ ).

This 60 -item questionnaire requires 4-mark answers in accordance with Lykert scale ( $1=\mathrm{I}$ do not usually do that, to $4=\mathrm{I}$ almost always do that). Its administration is individual, and the subject is asked to evaluate how many times s/he activates that specific coping process in difficult and stressful situations.

- the FACES III (Family Adaptability and Cohesion Evaluation Scale) [24, 25], to investigate mothers' perception of their own family functioning, during their child's treatment phase. It is a self-report tool made up by two series of items (20 items used to define the real family and other 20 items to define the ideal one) referring to the two great indicators of family functioning, the adaptability and cohesion, as 
fixed by Olson's model. This scale requires 5-mark answers in accordance with Lykert scale $(1=$ almost never to $5=$ almost always $)$. Very high levels of cohesion define the family functioning as characterized by the emotional enmeshment, which may lead to an emotional contagion among the family members. More specifically, each member may feel the same feeling of the other and no longer be able to have control on his/her own life individually. On the contrary, very low levels of cohesion refer to a family functioning based on the emotional detachment, which may lead to the lack of shared reciprocal empathy, useful for managing the critical events. It is a tool, whose original version by Olson (1985) was translated into Italian, which is characterized by good psychometric qualities such as the reliability of both internal consistency (total $r=0.68$ ) and test-retest ( $>0.80$ for dimension). Moreover, the scale has exhaustive levels of validity and good levels of internal coherence (correlation among the scales $=0.03$; correlation of each dimension with social desirability $=0)$.

With regards to the adaptability/flexibility dimension, very low levels may lead to rigidity of relationship between the family members in relation to roles, functions, and tasks. For instance, when a family member, typically the mother, cannot carry out her usual housekeeping, due to their child's treatment, none of the other family member-such as her son/daughter-will do it, unless s/he is requested to do. On the contrary, very high levels of flexibility may cause chaotic family functioning, so that their daily-life management will have neither shared rules nor orientation.

Furthermore, as for cohesion and adaptability levels, this instrument allows to detect three types of family functioning such as (1) the balanced functioning, as equilibrium between closeness and detachment among family members; (2) the intermediate functioning, that is typical of families who alternate moments of balanced and nonbalanced functioning; (3) the extreme functioning, characterized by an excessive closeness or detachment among the family members.

\section{Statistical Analysis}

The obtained data were analyzed through descriptive nonparametric statistics. In particular, the contingency coefficient $\left(\chi^{2}\right)$ was used to detect significant differences among indicators of functioning perception of both real and ideal family. Furthermore, the Friedman's test was used to analyze significant likely differences among the five studied coping strategies, whereas Spearman's $r$ coefficient was used to calculate the likely correlations between coping strategies typology and family functioning indicators (cohesion and real adaptability).

\section{RESULTS}

The data related to coping strategies (Fig. 2), gained by the administration of the COPENVI, show how the involved mothers were significantly characterized by positive aptitude, orientation toward problem, and request for social support $(F=99.8, \mathrm{~d} f=4$, $P<.01)$.

The data, obtained using the FACES III scale, show statistically significant differences among the levels of family's adaptability (rigid, structured, flexible, chaotic family) when it refers to both real $\left(\chi^{2}=13.29, \mathrm{~d} f=3, P=.004\right)$ and ideal $\left(\chi^{2}=11.52\right.$, $\mathrm{d} f=2, P=.003$ ) family functioning. Such data, in fact, stress the predominance of chaotic levels of functioning in both cases. No statistically significant differences are showed among the levels of cohesion (detached, separated, connected, enmeshed family) when it refers to both real $\left(\chi^{2}=12.3, \mathrm{~d} f=3, P=.006\right)$ and ideal $\left(\chi^{2}=12.58\right.$, $\mathrm{d} f=3, P=.006$ ) family functioning. Yet, it seems that the involved mothers had the 


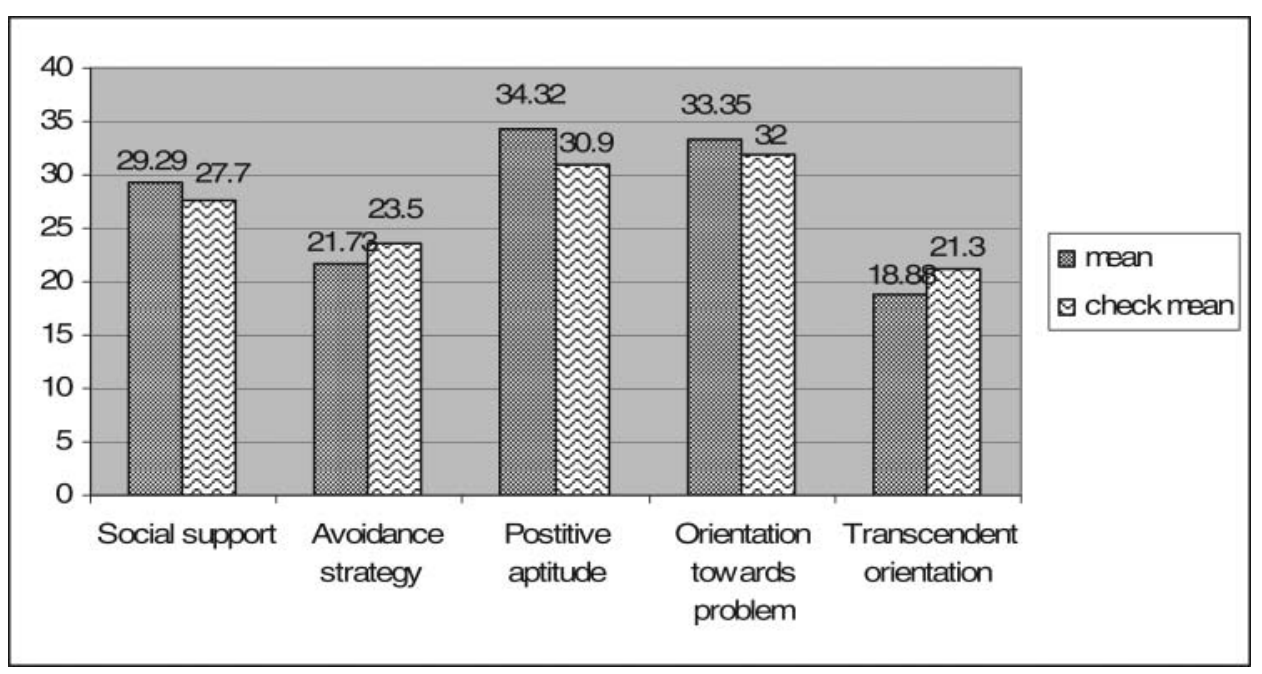

FIGURE 2 Mothers' coping strategies $(N=34)$.

tendency to feel the functioning of their real family, mostly characterized by detachment, whereas they wished the functioning of their ideal family characterized by enmeshment.

Finally, in relation to the likely correlations between typologies of coping strategies and indicators of real family functioning (Table 2), statistically significant positive correlations were only detected between adaptability and avoidance $(r=0.49, P<.01)$; adaptability and orientation toward problem $(r=0.36, P<.05)$; and between adaptability and transcendent orientation $(r=-0.04, P<.05)$.

\section{DISCUSSION}

The outcomes show the presence of some resources used by the mothers of children suffering from tumor, during the treatment phase. Such resources are of great interest for the quality of the relationship with health workers involved in the child's cure and care. In particular, one of them is the positive aptitude, as it is a specific emotional and cognitive coping strategy, which leads the mothers toward optimistic view of current situation. Hence, it would seem that these mothers try to have constructive, hopeful and faithful thoughts about their child's recovery, despite their awareness of all child's physical and psychological implications brought by the treatment.

This attitude would allow parents to bear the treatment course, and lead them to the therapeutic alliance with physicians, which is necessary for child's cure [9].

TABLE 2 Correlations Between Coping Strategies and Real Family Functioning Factors (Spearman's r Coefficient)

\begin{tabular}{lcc}
\hline & Cohesion & Adaptability \\
\hline Social support & 0.27 & -0.14 \\
Avoidance & 0.18 & 0.14 \\
Positive aptitude & 0.18 & 0.18 \\
Orientation toward problem & $0.49^{*}$ & $0.36^{* *}$ \\
Transcendent orientation & -0.004 & $-0.40^{* *}$ \\
\hline
\end{tabular}

${ }^{*} P<.01$ (two-tailed).

${ }^{* *} P<.05$ (two-tailed) 
The orientation toward problem skill, which is mothers' attitude to plan activities aimed to handle the situation, is proved to be another remarkable strategy coping. The predominance of such coping strategy emphasizes the mothers' attempt to maintain their attention on the problems that are peculiar to the treatment phase, and to request information to solve these problems. It is this coping strategy that may allow mother to continuously assess the stressor situations, and to detect the possibility of change in external environment or even inside [26].

This mothers' aptitude is important to build with health workers a relation based not only on compliance, as acceptation of the therapeutic protocol, but also on the adherence, which involves the conscious adhesion. Furthermore, such data underline how important can be that medical staff provides parents a clear and effective communication about conventional and nonconventional therapies, and their likely relevant implications. The acquired information, in fact, can guide and support them, in developing proper adaptive behaviors, useful for coping the critical event.

It is the clarity of communication about the value of the treatment, and the adequacy of the provided information about both the tumor treatment in general, and the specific therapeutic course suggested for their child, that may decrease the risk of parents' refusal. In fact, parents who are not provided with proper information, may decide not to comply the treatment of choice, which would lead to fail the collaboration with medical staff and to stop the treatment [27]. Moreover, parents who take part in their child's cure course, may reduce their feeling of powerlessness and uselessness.

Finally, the social support is another coping strategy that has prevailed in the involved mothers. It would seem, in fact, that they have been able to ask their partner, or other relative and even medical staff, for help in order to get emotional control, information and support to practically manage the critical situation. Such an outcome shows that parents of children suffering from tumor, feel the role of physicians a priority to take care of their children, as if they were real caregivers. Undoubtedly, their request for support and empathy to physicians may be very important for the therapeutic alliance, even though, at the same time, it implies an "emotional burden" which, if it is not properly managed, can lead to the burnout condition [28].

As for the results concerning the perception that the mothers have had of their family functioning during the treatment phase of their child, some interesting aspects for the interaction between physicians and families are stressed. The mothers have described their family functioning in extreme terms, with regard to both cohesion and adaptability. It would seem, in fact, that families, during their child's complex phase of treatment, had the tendency to function in enmeshed way-when all family's members are too close-and, at the same time, in chaotic way, so that they were not able to modify functions and habits to better manage the new needs arisen from treatment. Furthermore, it would seem that mothers wished to have such kind of family functioning, when they were asked to describe their both real and ideal family functioning. It is clear that, if a functioning characterized by cohesion (enmeshment) lasts during the whole path of illness, it can be dysfunctional for the adaptive management of risk condition and the relationship with child. Yet, such tendency to be enmeshed can be considered as a resource for families, whenever it is turned into the ability of all family members to support each other to manage the critical events. Since such closeness can reduce the likely feeling of loneliness of mothers, who usually are the preferred people experiencing the daily cure in hospital together with their child, it may ease their active involvement in their child's therapeutic path planned by physicians.

The outcome relating to the significant correlation between the mothers' perception of their family functioning and coping strategies, it is also very important. It would 
seem that, in families characterized by emotional closeness, reciprocal support and capability of adjustment to meet the new needs of the treatment phase, mothers have been able to manage and analyze problems and have not got disheartened when facing difficulties. It would also seem that, the more families are characterized by adaptability, the less mothers resort to supernatural entities (e.g., resorting to faith) as a coping strategy.

Such outcomes, then, would seem to show how important is that medical staff

- meet the whole family, to reinforce the importance of closeness among family members when faced with the difficulties arisen by the treatment phase;

- understand the difficulties that they meet or they could meet;

- help them in finding out what of their functioning needs to be modified in order to properly take care of their child and secure him/her quality life.

So, it is of prime importance that physicians plan specific time to be spent in meetings and talking to child's parents, to guide them in realizing what is happening or what will happen, to support them for the decisions to be made, etc. [18]. Doing so, parents will be an integral part of the sustained, not-episodic and communicative process that should characterize the long path, from the diagnosis up to treatment, toward the likelihood of recovery or, however, the child's life quality [18].

All the considerations, arisen from the data of the study, would seem to suggest some specific criteria that can be useful for the ethic management of the interaction with families, which has a prime role in determining the quality of child's treatment path. This active involvement of parents implies that they are asked to take some responsibilities upon themselves, concerning the often-complex choices requested by the path.

The criteria indicated below are related to the management of the communication with families and the taking charge of their special needs

- clarity and sincerity of communication in every stage of the treatment path. It implies, first of all, that physicians whenever provide information, clarifications and proposals to families, have always to consider what knowledge, representations, likely prejudices, expectations, etc. parents have about their child's illness and its relevant treatment. Namely, it would mean to collect the informal knowledge concerning the child's illness and treatment that families have as a result of personal and/or others' experience, and based on an autonomous research of information (i.e., Internet). Such physicians' behavior may foster in parents a clear, aware comprehension without any misunderstandings about the specific situation. With regards to the sincerity, physicians should provide a realistic communication, neither too optimistic, nor too pessimistic or hopeless. It would mean to unveil family the likely difficulties and criticalities related to the treatment that can be managed together. Finally, since the data of the study show the families' coping skills, it is to be remarked the importance of the involvement of all the families' components (parents' and siblings' child), at different stages of the treatment, though at different times and with different way.

- Acknowledging and granting the families' needs at emotional, rational, and ethic levels. It is of prime importance that physicians be empathetic with each member of a family when they are informed about the treatment and the new procedures to be followed, listen at and control them, or suggest them other form of supports such as a psychologist. Physicians should also focus on what information can be 
misunderstood and what explicit or even not explicit doubts can arise, besides trying to know families' cultural background.

\section{CONCLUSION}

The data of the study would seem to confirm the literature of the field, with regards to the factors called moderators of adjustment of parents of children with tumor [11, 12, $15,29]$. To be more precise, the data of the study would seem to confirm the literature of the field, with regards to some of the factors meant as moderators of adjustment of parents of children suffering from tumor $[11,15,15,12,29]$. To be more precise, it focuses on the predominance of positive aptitude as coping strategy, used by the mothers involved in this study, even more frequently than the normative sample does. It is, in fact, the strategy most used by the parents of children suffering from tumor, because it would seem to help them during the adjustment process to risk condition [30]. The datum related to positive aptitude, which has been obtained through the sample of the study, is coherent and consistent with the contributions of the field which underline that parents' positive aptitude could be a sort of healthy denial and, at cognitive level, a selective information processing $[2,1]$.

With regards to social support as another coping strategy, it has to be said that, even though it is not the main strategy used by the mothers of the sample, however, it is the positive aptitude and orientation toward problem with a mark higher than that of normative sample. It is a datum that would seem to confirm the value of social support as predictive factor of good adjustment of parents of children with leukemia $[11,13,31$, 32].

Yet, the outcomes of the study are not consistent with those scientific contributions that have underlined the importance of resorting to pray and religion, as an effective coping strategy very used by parents of children suffering from tumor, especially during the acute stage of illness $[33,19]$. The data, in fact, show that the mothers involved in the study would seem to rarely resort to such a strategy (transcendental orientation). Furthermore, the correlation between this coping strategy and the family adaptability, would seem to suggest that restoring to the an intimist dimension, linked to only one parent (only mothers, in this case), may prevent the reorganization of family system from successfully meeting the needs required by the treatment phase.

The study is also not consistent with other studies of the field, in relation to the factors that are moderators of parents' adjustment connected with peculiarities of the family system. It has considered, in fact, mothers' perception of the functioning of the whole family system, rather than the dimension of marital satisfaction usually investigated by other studies [11,32], where the perception of such factor is shown in extreme terms (extreme entanglement and chaos). This outcome would suggest a closer examination. It is true that mothers' perception of an extreme entanglement among the members of her family, during the acute phase of illness, could be useful for her adjustment; it is also true, however, that this entanglement could be a risk when it lasts even during the chronic phase, since it could distress each family member and, hence, be no longer useful for facing the specificity of the new phase of illness.

\section{Declaration of Interest}

The authors declare that they have no conflict of interests. The authors declare that they have full control of all primary data and they agree to allow the journal to review their data if requested. 


\section{REFERENCES}

[1] Biondi M, Costantini A, Grassi L. La mente e il cancro: insidie e risorse della psiche nelle patologie tumorali. Roma: Il Pensiero Scientifico; 1995.

[2] Van-Dongen-Melman JE, Sanders-Wodustra H. Psycholgical aspects of childhood cancer: a reviewof the literature. J Child Psychol Psychiatry. 1986;27(2):145-189.

[3] Sawyer M, Antoniou G, Toogood I, et al. Childhood cancer: a two year prospective study of the psychological adjustment of children and parents. J Am Acad Child Adolesc Psychiatry. 1997;36(12):1736-1743.

[4] Sawyer M, Antoniou G, Toogood I, et al. Childhood cancer: a 4 year prospective study of the psychological adjustment of children and parents. J Pediatr Hematol Oncol. 2000;22(3):214-220.

[5] Van Dongen-Melman JEWM, Pruyn JFA, De Groot A, et al. Late consequences for parents of children who survived cancer. J Pediatr Psychol. 1995;20:567-586.

[6] Doka KJ. Living with Life-Threatening Illness: a Guide for Patientts, their Famiglie and Caregivers. San Francisco, CA: Jossey-Bass; 1993.

[7] Patterson MJ, Holm KE, Gurney JG. The impact of childhood cancer on the family: a qualitative analysis of strains, resources and coping behaviours. Psycho-Oncology. 2004;13:390-407.

[8] Patterson JM, Gatwick AW, Bennett CF, et al. Social support in families of children with chronic conditions: supportive and non supportive behaviours. J Dev Behav Pediatr. 1997;18(6):383-391.

[9] Masera G, Spinetta JJ, Jankovic M, et al. Guidelines for a therapeutic alliance between families and staff: a report of the SIOP working committee on psychosocial issues in Pediatric Oncology. Med Pediatr Oncol. 1998;30:183-186.

[10] AIEOP. Tutti bravi. Psicologia e Clinica Del Bambino Portatore di Tumore. Milano, TX: Raffaello Cortina; 1998.

[11] Hoekstra-Weebers JEHM, Jaspers JPC, Kamps WA, et al. Psychological adaptation and social support of parents of pediatric cancer patients: a prospective longitudinal study. J Pediatr Psychol. 2001;26(4):225-235.

[12] Goldbeck L. Parental coping with the diagnosis of childhood cancer: gender effects, dissimilarity within couples, and quality of life. Psycho-Oncology. 2001;10(4):325-335.

[13] Manne S, Duhamel K, Redd WH. Association of psychological vulnerabilità factors to post-traumatic stress symptomatology in mothers of paediatric cancer survivors. Psycho-Oncology. 2000;9:372-384.

[14] Dockerty JD, Willimas SM, McGee R, et al. Impact of childhood cancer on the mental health of parents. Med Paediatr Oncol. 2000;35:475-483.

[15] Sloper P. Predictors of distress in parents of children with cancer: a prospective study. J Pediatr Psychol. 2000;25(2):79-91.

[16] Norberg LA, Lindblad F, Bornan KK. Coping strategies in parents of children with cancer. Soc Sci Med. 2005;60(5):965-975.

[17] Patistea E. Description and adequacy of parental coping behaviours in childhood leukaemia. Int J Nurs Stud. 2005;42(3):283-296.

[18] Spinetta JJ, Jankovic M, Masera G, et al. Optimal care for the child with cancer: a summary statement from the SIOP working committee on psychosocial issues in Pediatric Oncology. Pediatr Blood Cancer. 2009;52(7):904-907.

[19] Schneider MA, Manell RC. Beacon in the storm: an exploration of the spirituality and faith of parents whose children have cancer. Issues Compr Pediatr Nurs. 2006;29(1):3-24.

[20] Rajajee S, Ezhilarasi S, Indumathi D. Psychosocial problems in families of children with cancer. Indian J Pediatr. 2007;74(9):837-839.

[21] Olson DH. Circumplex model VII: validations studies and faces III. Fam Process. 1986;25:337-351.

[22] Sica C, Magni C, Ghisi M, et al. Coping Orientation to Problems Experienced-Nuova versione iataliana (COPE-NVI): uno strumento per la misura degli stili di coping. Psicoterapia cognitiva e comportamentale. Edizioni Erickson, Trento. 2008;14(1):27-53.

[23] Carver CS, Scheier MF, Weintraub JK. Assessing coping strategies: a theoretically based approach. J Pers Soc Psychol. 1989;56(2):267-283.

[24] Olson DH. Faces III, F.S.S. Minnesota, MN: St. Paul; 1985.

[25] Galimberti C, Farina M. FACES III. Uno Strumento per la Ricerca e L'osservazione Clinica Della Famiglia. Milano, TX: Vita e Pensiero; 1992.

[26] Compas BE, Boyer M. Coping and attention: implications for child health and pediatric conditions. J Dev Behav Pediatr. 2001;22:1-11.

[27] Spinetta JJ, Masera G, Eden T, et al. Refusal, non-compliance, and abandonment of treatment in children and adolescents with cancer. A report of the SIOP Working Committee on Psychosocial issues in Pediatric Oncology. Med Pediatr Oncol. 2002;38(2):114-117.

[28] Spinetta JJ, Jankovic M, Ben Erush MW, et al. Guidelines for the recognition, prevention and remediation of burnout in health care professionals participating in the care of children with cancer. A 
report of the SIOP Working Committee on Psychosocial issues in Pediatric Oncology. Med Pediatr Oncol. 2000;35(2):122-12.

[29] Axia V (a cura di). Elementi di Psico-Oncologia Pediatrica. Roma: Carocci Faber; 2004: 277-300.

[30] Hardy MS, Armstrong FD, Routh DK, et al. Coping and communication among parents and children with human immunodeficiency virus and cancer. J Dev Behav Pediatr. 1994;15(suppl):49-53.

[31] Kupst MJ, Schulman J. Long term coping with paeditric leukaemia. A six year follow up study. $J P e-$ diatr Psychol. 1988;13:7-22.

[32] Magni G, Messina C, De Leo D, et al. Psychological distress in parents of children with acute lymphatic leukaemia. Acta Psychiat Scand. 1983;68:297-300.

[33] Cayse LN. Fathers of children with cancer: a descriptive study of their stressors and coping strategies. J. Pediatr. Oncol. Nurs. 1994;11(3):102-108. 